\title{
Brokers en una red de venta de derechos de uso de tierra: Valle de Rengo, Chile, 1832-1860 1
}

Brokers In A Land Use Rights Sale Network. Rengo Valley, Chile, 1832-1860

Víctor Brangier*

Alexis Matheu**

\section{RESUMEN}

El artículo analiza una red de venta de derechos de uso de tierra en un valle de Chile, en periodo de formalización de derechos (1832-1860). Se examina la conducta de brokers desde la noción Agujeros Estructurales, proveniente del Análisis de Redes Sociales. Mediante el estudio de contratos de compra y venta y de catastros agrícolas, se deduce que los brokers privilegiaron comprar tierras mejor valuadas a vendedores de baja participación en la red. La estrategia rindió frutos patrimoniales en pocos brokers vinculados a la administración pública local. Se proyecta la relevancia del hallazgo para la literatura centrada en el ciclo: formalización de derechos/ desposesión campesina.

Palabras clave: análisis de redes sociales - derecho a uso de tierras - Chile.

\section{Abstract}

The article analyzes a land use rights sale network in a valley of Chile, in the period of formalization of rights (18321860). The behavior of brokers is examined from the notion of Structural Holes from the Social Network Analysis. Purchase and sale contracts and agricultural cadasters are studied, deducing that the brokers favored buying high value land from sellers with low participation in the network. The strategy paid off in a few brokers linked to the local public administration. The relevance of the finding is projected for the literature focused on the cycle: formalization of rights / peasant dispossession.

Keywords: Social Network Analysis Land use right - Chile.

\footnotetext{
* Universidad Bernardo O 'Higgins, Santiago, Chile. vmbrangi@gmail.com <https://orcid.org/00000003-3583-2221>

** Universidad Bernardo O 'Higgins, Santiago, Chile. alexis.matheu@ubo.cl <https://orcid.org/00000002-7859-9039>
} 
Por lo general se ha establecido que el proceso de formalización de derechos sobre la tierra en América Latina se expandió desde 1850. El ciclo había comenzado con la conminación estatal para formalizar las posesiones de facto de este bien ante oficinas nacionales o locales que se crearon con este propósito. Una segunda etapa continuó con la desposesión de las tierras que ocupaban informalmente los campesinos desde mucho tiempo o con el arrebato de las tierras baldías que los colonos habían vuelto productivas. La desposesión se había originado en las ventajas con las cuales contaban los grandes especuladores de materias primas y las familias terratenientes, en su competencia contra colonos y campesinos por la formalización de tierras lucrativas. El trayecto se había cerrado con la incorporación de estos actores expropiados en calidad de trabajadores dependientes en las grandes propiedades agrícolas recién formalizadas. ${ }^{2}$

En la última década, la literatura ha revisitado este ciclo problematizando el concepto de propiedad. Ha quedado al descubierto que, previo a (y durante) la formalización jurídica de las posesiones, coexistían formas plurales y legítimas de uso, goce y disposición de la tierra. ${ }^{3}$ Convivían regímenes propietarios híbridos que distaban del paradigma liberal de propietarios con potestad excluyente sobre la tierra inscrita bajo su nombre. ${ }^{4}$ Recurriendo al enfoque histórico-jurídico que ha puesto en evidencia la multiplicidad de concepciones propietarias, se cuenta con un prisma que ha cuestionado el paradigma evolutivo del avance de la "propiedad imperfecta" a la "propiedad perfecta". La discusión ha minado el relato lineal ortodoxo que proponía el reemplazo de una forma de tenencia ineficiente y sin propiedad privada por un régimen productivo y con propietarios reconocidos (Congost, Gelman, y Santos, 2017). La problematización histórica del concepto de propiedad ha permitido, en cambio, visualizar que sobre un mismo paño de tierra se negociaban y repartían distintos derechos a uso, goce y disposición que involucraban a diversas gentes, familias o instituciones. El derecho a la tierra y a sus frutos, independiente de su inscripción o no en registros locales o estatales, debía disputarse en el plano de las relaciones sociales. Este énfasis en la negociación y tensión entre quienes se repartían los derechos a este bien ha exhortado al estudio de la circulación de información variada entre actores que recurrían a sus distintas concepciones propietarias, al pretender cuotas de acceso a la tierra. ${ }^{5}$

El presupuesto de la negociación y disputa social sobre información plural relativa al derecho a la tierra permite enriquecer la mirada en torno al ciclo formalización de derechos/desposesión campesina. El enfoque habilita el examen de formas "para-propietarias" (Grossi, 1992, p. 70), asumidas por actores 
carentes de derechos formales o desposeídos de los mismos, quienes apelaron a distintas interpretaciones sobre sus derechos a la tierra. ${ }^{6}$ Atendiendo esta oportunidad analítica, este artículo problematiza este campo de negociaciones sociales sobre interpretaciones plurales, específicamente respecto a los derechos de uso sobre la tierra. Las siguientes páginas abordan un caso de estudio: una red de venta de tierras en el valle de Rengo, en la zona central de Chile durante la primera mitad del XIX. Interesa detenerse en los gestores o brokers de información variada, quienes, mediante la administración de múltiples concepciones propietarias, aspiraron a ventajas en la adquisición patrimonial. La investigación examina la venta de derechos de uso sobre la tierra entre agricultores de rentabilidad media y baja, en este valle entre 1832 y 1860 . La interrogante central se asocia al modo en que los sujetos impulsaron sus intereses, instalándose en posiciones ejes de un circuito de compra y venta y administrando concepciones propietarias plurales. Como objetivo general se pretende analizar la conducta asumida por actores instalados en posición de brokers en la red de ventas. Complementariamente se busca evaluar la repercusión que tuvieron esas conductas en la evolución patrimonial de esos actores a lo largo del periodo.

El espacio escogido corresponde a una zona de agricultura triguera, ubicado a 120 kilómetros al sur de Santiago y posicionado en la región geográfica chilena reconocida como "valle central". El área fue atravesada por el camino principal longitudinal y fertilizada por el Río Claro y sus afluentes. El valle tuvo como sede administrativa la villa y municipio de Rengo, insertos en el Departamento de Caupolicán, subdivisión de la Provincia de Colchagua. Después de la provincia de Santiago, Colchagua concentró la mayor densidad poblacional del país en el siglo XIX (Brangier, 2019). Se trató de un escenario de desarrollo del latifundio agrícola y ganadero desde el siglo XVIII, pero con presencia significativa de pequeñas economías campesinas que vendían sus productos a las haciendas y a los incipientes focos urbanos de la provincia (Cáceres, 2007). Sobre esta área, como se explicará en la siguiente sección, existe más de un millar de documentos de compra y venta de derechos de uso. La serie documental comienza en 1832 y culmina en 1860 . El periodo coincide con décadas que han sido consideradas por la literatura como representativas de un alza en el valor de la tierra, de recuperación económica tras las batallas de independencia y de desposesión campesina en favor de la Gran Hacienda triguera conectada progresivamente con mercados internacionales (Llorca y Navarrete, 2017; Salazar y Pinto, 2002). Estos factores complementaron el impulso por identificar oficialmente el tamaño y rentabilidad de cada predio 
(sobre todo con fines de recaudación fiscal) y formalizar los derechos de cada propietario. El primero de estos procesos se llevó a cabo desde la profesionalización de la agrimensura y la aplicación de catastros agrícolas (Álvarez, 2014). El segundo, mediante la creación de un Registro Conservatorio de Bienes Raíces desde 1857. La institución cristalizaba las ideas del jurista Andrés Bello en su Código Civil de 1855, en que estableció la exigencia de crear la institución para reconocer como propiedad solo los bienes raíces inscritos (Estado de Chile, 1882, p. 101).

Primero, se explica el método con el que se procesó la información. En este apartado se define la noción de Agujeros Estructurales proveniente del Análisis de Redes Sociales y las tres variables que sirvieron para el cumplimiento de los objetivos. Después se expondrá los resultados de las tres variables y su cruce según el análisis multivariado. Allí se identificarán los actores que, a lo largo de estos años, emprendieron la compra de derechos de uso de las tierras mejor valuadas, transando preferentemente con actores de baja experiencia en este tipo de transacciones. En esta sección se detectó un grado de relación moderada entre quienes asumieron la estrategia señalada y su progresión patrimonial. Por último, se proyecta la contribución del caso de estudio a la discusión sobre el periodo de formalización de derechos a la tierra y crisis en el acceso social a la tierra, con especial énfasis en la literatura sobre Chile.

\section{MÉTOdO: ANÁLISIS DE REDES SOCIALES}

Y BROKERS EN AGUJEROS ESTRUCTURALES

El estudio utiliza las herramientas del Análisis de Redes Sociales y la noción de Agujeros Estructurales (AE, en adelante). En la revisión del concepto de capital social, el sociólogo Ronald Burt concluyó que se le ha caracterizado como metáfora de ventaja en medio de interacciones sociales. Las personas intercambiarían recursos (información, influencias, bienes, etc.) en persecución de sus intereses. En la contraposición de esfuerzos, obtendrían más ventaja quienes logren mejores posiciones en la estructura relacional (Burt, 2001). La propuesta de Ronald Burt se inserta en el debate sobre las fuentes de obtención de capital social y se inclina por las ventajas obtenidas por quienes median en regiones de baja conexión en las redes sociales. ${ }^{7}$ En este sentido, el autor acuña la noción de $\mathrm{AE}$, caracterizados como espacios de la red social con ausencia de lazos entre segmentos. Los actores instalados en esos escenarios ac- 
tuarían como puentes o broker. Se trataría de una posición privilegiada de adquisición de capital social: el broker obtendría información variada proveniente de cada subgrupo, controlaría los flujos de transferencia de esa información entre las distintas riberas de la red y asumiría liderazgo al gestionar los contactos que, sin su presencia, estarían desunidos (Burt, 2010).

El Análisis de Redes Sociales y la teoría del broker se centran en la circulación y gestión de información variada en un grupo determinado. Por esta razón, este marco teórico-metodológico se ha escogido para abordar el problema de este estudio: las negociaciones sociales en torno a interpretaciones variadas de derechos de uso sobre la tierra. La investigación examina la generación de $\mathrm{AE}$ en la red social que se ha descrito más arriba. El escrutinio se basa en 1.526 contratos de venta entre privados, conservados en siete volúmenes del Fondo de Notarios de Rengo (FNR, en adelante) del Archivo Nacional de la Administración de Chile (Arnad, en adelante). El primer criterio de selección documental propició la búsqueda de agricultores de recursos medios y bajos. Para ello, se cotejó la información de los nombres presentes en los contratos notariales de venta con los nombres de tributantes del catastro agrícola de 1833/1834. Más adelante se caracterizará este documento específico. Por el momento es necesario puntualizar que este catastro gravó la producción agrícola destinada al mercado. Aquellos agricultores que sobrepasaban la barrera de 100 pesos de producción anual (la cifra excedía el promedio de avalúos del total de tributantes) se consideraron en esta investigación como grandes propietarios y sus transacciones se excluyeron de la muestra notarial seleccionada.

El segundo criterio se selección documental permitió escoger contratos en que se especificara la venta de "derechos de uso", privilegiando esta expresión por sobre otras presentes en el Fondo, como "venta de tierras o "venta de cuadras". El examen de las fuentes permitió detectar que sobre aquellas formas de transferencias hubo interpretaciones sociales más variadas sobre el significado de la sustancia transada que sobre estas últimas. El criterio permitió levantar una muestra en que coexistieron concepciones propietarias heredadas de la cultura jurídica del derecho castellano e indiano, con nociones propietarias emergentes en el periodo en estudio. En el mundo colonial hispanoamericano, los tres derechos que integran el dominio: derechos de uso, goce y disposición, se encontraban "desmembrados" entre actores que debían interactuar para disputar o negociar la actualización de sus prerrogativas sobre la misma porción de tierra. ${ }^{8}$ En el siglo XIX hubo una tendencia discursiva y legislativa a reunir esos tres poderes en un solo titular del bien, atendiendo las ansiedades liberales por construir mercados de tierra con propietarios defini- 
dos y las necesidades de recaudación fiscal vía tributos agrícolas (Palacios, 2011, p. 67). Este trasfondo sustentó el proceso de formalización de derechos de uso de la tierra que se ha descrito anteriormente. Los contratos escogidos develan la convergencia de concepciones propietarias de distintos tiempos y legados en las prácticas de venta de "derechos de uso". Para algunos, esta expresión implicaba la compra solo del uso de la tierra, excluyendo el goce íntegro de sus productos. ${ }^{9}$ Para otros, la compra significaba la adquisición plena y soberana del bien con exclusión de derechos de otras personas. ${ }^{10}$

El estudio consideró tres variables. Las dos primeras fueron medidas mediante el software UCINET, que permite abordar variables de redes y construir grafos, a partir de bases de datos que contengan información relacional. La base de datos integró 1.526 filas correspondiente con cada transacción de venta. La información se desplegó en tres columnas que reflejaron los nombres del vendedor, del comprador y el promedio del valor cuadra de la transacción. Las dos variables medidas con UCINET fueron In-degree y Effective Size. El primero expresa la cantidad de lazos que recibe un actor (un nodo) en la red. En este caso, la cantidad se vincula con el promedio del valor cuadra que recibió cada nodo (participando, por tanto, como comprador). Este cálculo permitirá aproximarse a una identificación de quienes exhibieron una conducta de compras de las tierras mejor valuadas en la red a lo largo del periodo. Por otro lado, el indicador Effective Size es una de las herramientas más utilizadas en la detección de AE y de brokers en el Análisis de Redes Sociales. Identifica el número de contactos directos de un nodo (denominado EGO) en una red, restando el promedio de los contactos directos que esos nodos mantienen dentro de la red de EGO (evitando contar los vínculos con EGO). El resultado es una cifra neta proporcionalmente directa a la capacidad de mediación de EGO entre sus contactos y entre ellos y el exterior (mientras más alta la cifra, mayor capacidad de intermediación de EGO) (Borgatti; Everett; Johnson, 2013, p. 289).

La tercera variable se denominó "Progresión Patrimonial" y se obtuvo desde tres catastros agrícolas contemporáneos. El gobierno produjo catastros en los años 1833/34, 1837/1838, 1852 y 1855, registrando el avalúo de la producción anual que las propiedades agrícolas destinaban al mercado. Tras el cálculo se estableció un tributo del $3 \%$ y posteriormente del $4 \%$ por predio (Álvarez, 2014). En esta investigación se escrutó la información de la renta anual de los brokers, identificados en las dos variables anteriores, en los catastros de 1833/34, 1852 y 1855. Este examen permitió observar el grado de Progresión Patrimonial por nodo en dos décadas y deducir su vinculación con la estrategia de construcción de $\mathrm{AE}$ y gestión de información variada sobre la compra de derechos de uso de 
la tierra mejor valuada. Las tres variables se vincularon mediante un análisis multivariado expresado en un gráfico exploratorio HJ-Biplot que permite representar geométricamente individuos, objetos o subpoblaciones de una muestra mayor, respecto a un conjunto de variables observables (Galindo, 1986).

\section{Resultados}

La red estuvo compuesta por 1.526 vínculos que relacionaron a 1.659 actores. La estructura manifestó un componente principal de 890 nodos y 988 lazos, correspondiendo al 52,6\% de los actores y al 64,7\% de los vínculos. La relevancia del componente principal exhortó analizar sus principales ejes. Se incluyeron en la medición los promedios del valor por cuadra de las transacciones. El resultado corresponde con la Figura 1 que devela el componente principal valuado según el promedio indicado. A través de UCINET, se alteró proporcionalmente el grosor de los lazos según ese valor: a mayor promedio mayor grosor.

Figura 1 - Componente principal (valuado por promedio valor cuadra). Red de venta de derechos de uso de tierras. Rengo, Chile, 1832-1860

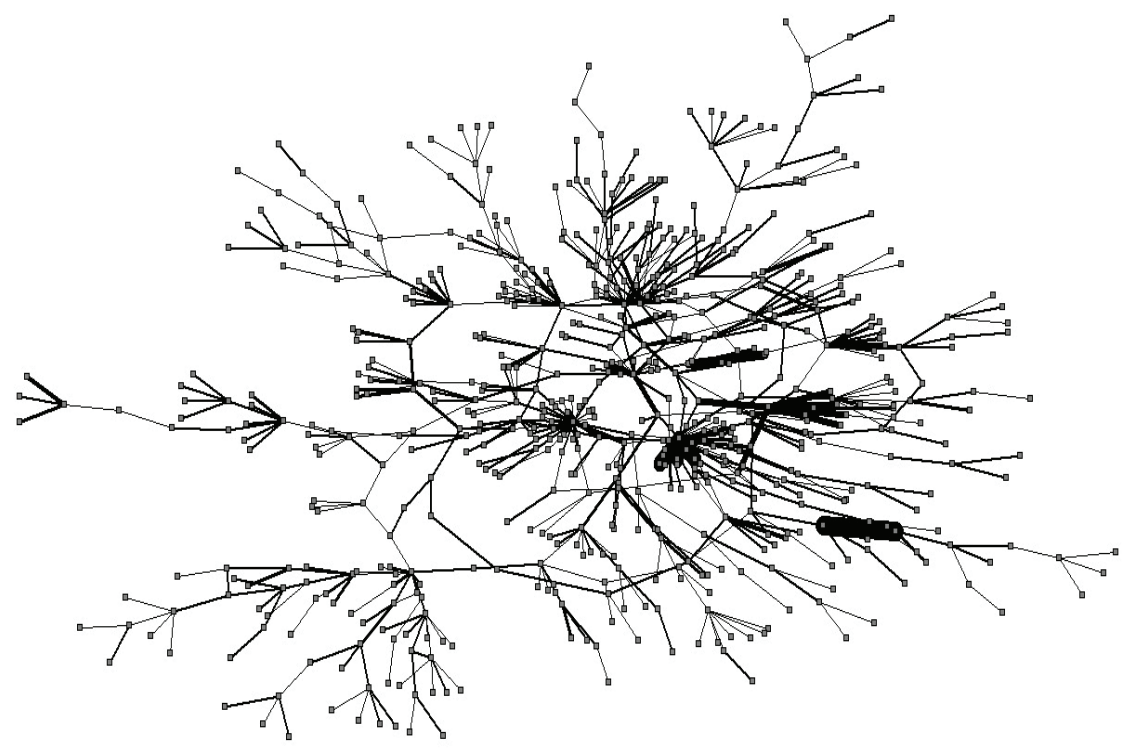

Fuente: (ARNAD, FNR, volúmenes 19, 21-26, 28-39, 44-50). 1.526 documentos de venta de derechos de uso de tierras. Procesado en UCINET versión 6.658. 
La Figura 1 devela la concentración de los mayores volúmenes de las medias en regiones específicas del centro de la estructura. Es posible visualizar que mientras los actores se encontraban más y mejor relacionados al conjunto, se aproximaban a mayores precios de venta. En la medida que aquellos ocupaban una posición más periférica, contaban con probabilidades menores de vender o comprar derechos de uso más caros. En este sentido, la Figura 1 insta a indagar en los nodos que, a la vez que se constituyeron en articuladores del conjunto, vehiculizaron la movilidad de los derechos a las tierras mayormente valuadas.

La documentación notarial permite deducir que las tierras de mayor valor por cuadra en el valle de Rengo cumplían con características comunes que las convertían en foco de atracción para los compradores con mayor proyección de expansión en la economía agrícola triguera. Generalmente se trataba de terrenos planos con acceso a cursos de agua y a caminos. En ocasiones se especifica que los compradores gozarían de "derechos a cerro" o que las cuadras incluían "todo lo edificado y plantado". Adicionalmente, la proximidad a la villa de Rengo, capital administrativa del Departamento de Caupolicán, también elevaba el valor, sobre todo si se ubicaban en el paño norte y nororiente de la villa que rodeaba el Río Claro. ${ }^{11}$ La Figura 2 ofrece una demarcación aproximada del valle de Rengo, en medio de la provincia de Colchagua en la zona central de Chile.

Figura 2 - Valle de Rengo, zona central de Chile, siglo XIX

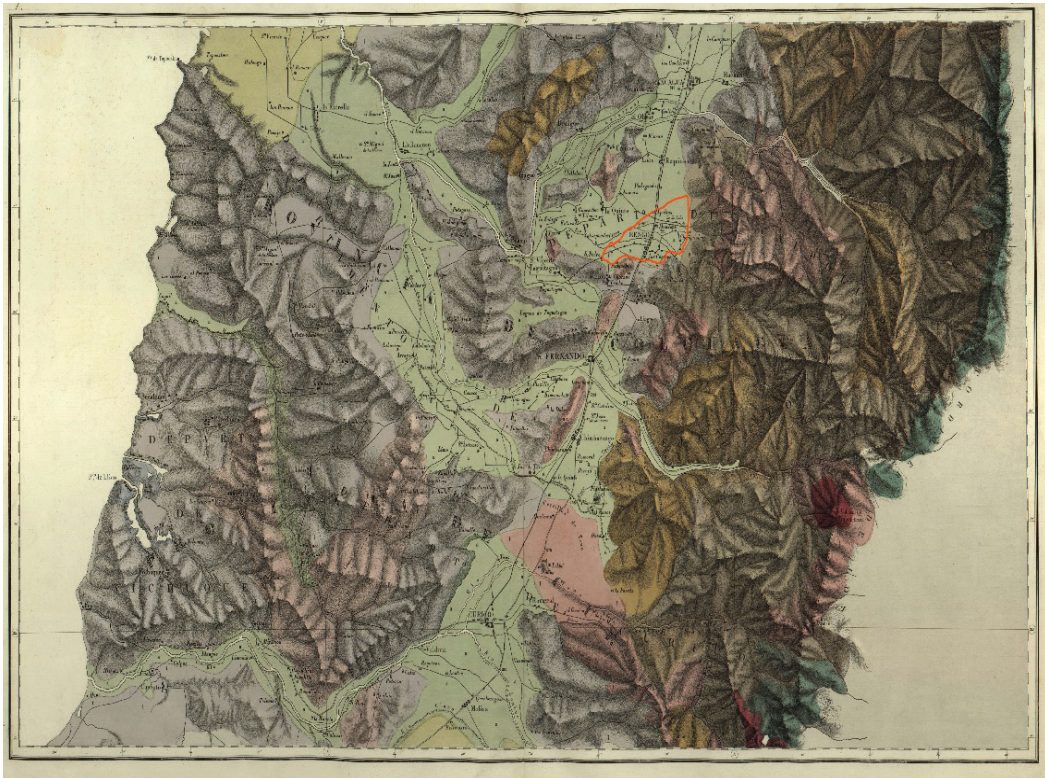

Fuente: (Pissis, 1873). Imagen editada por los autores de este artículo. 
¿Quiénes eran estos actores que se posicionaron en los ejes vertebrales de la red y que aspiraron a los derechos de uso de las tierras mejor valuadas? Para avanzar sobre esta interrogante, mediante UCINET se identificó a los actores que se concentraron en torno a las transacciones con los promedios de venta más altos. Se seleccionó el indicador In-degree para rescatar los nodos que compraron tierras con mayor promedio de valor cuadra. El Cuadro 1 expone los nombres de los nodos instalados en los 20 primeros lugares y los respectivos promedios valor cuadra que pagaron en cada transacción.

Cuadro 1 - 20 primeros nodos según In-degree.

Red de venta de derechos de uso de tierras. Rengo, Chile, 1832-1860

\begin{tabular}{|l|c|}
\hline \multicolumn{1}{|c|}{ Nodo } & $\begin{array}{c}\text { Promedio valor cuadra } \\
\text { por compra (pesos) }\end{array}$ \\
\hline 1.- Francisco Trujillo & 1.613 \\
\hline 2.- Juan José Mena & 1.200 \\
\hline 3.- Francisco Rivas & 875 \\
\hline 4.- León Ramírez & 605 \\
\hline 5.- Valentín Valuarte & 580 \\
\hline 6.- José Latorre & 552 \\
\hline 7.- José Morales & 476 \\
\hline 8.- Juan Molina & 439 \\
\hline 9.- Simón Valenzuela & 385 \\
\hline 10.- Lorenzo Banda & 315 \\
\hline 11.- Francisco Silva & 302 \\
\hline 12.- Valentín Madariaga & 288 \\
\hline 13.- Pedro Jasnavar & 275 \\
\hline 14.- Felipe Contreras & 272 \\
\hline 15.- Pedro Lorca & 239 \\
\hline 16.- Asencio Medina & 217 \\
\hline 17.- Ramón Jiménez & 217 \\
\hline 18.- Cecilio Alarcón & 215 \\
\hline 19.- Santiago Bosa & 214 \\
\hline 20.- Bernardo Ávila & 202 \\
\hline
\end{tabular}

Fuente: (ARNAD, FNR, volúmenes 19, 21-26, 28-39, 44-50). 1.526 documentos de venta de derechos de uso de tierras. Procesado en UCINET versión 6.658. 
El Cuadro 1 devela que el promedio del valor cuadra que pagaron en cada transacción estos 20 actores (474 pesos) quintuplicó al promedio del valor cuadra que se encontró en la red global $(87,12)$. Para avanzar un segundo grado en la identificación de los nodos que se posicionaron en ejes estratégicos de la red, se midió la ubicación de los actores en AE. A través de UCINET se abordó la variable Effective Size con el fin de pesquisar a quienes se vincularon con nodos débilmente conectados a la estructura. $\mathrm{O}$ en otros términos, para observar a los integrantes de la red que privilegiaron transar con actores que figuraron en escasas transacciones. Los resultados se encuentran en el Cuadro 2, donde se seleccionaron los 20 nodos con mayores valores absolutos.

Cuadro 2 - 20 primeros nodos según Effective Size. Red de venta de derechos de uso de tierras. Rengo, Chile, 1832-1860

\begin{tabular}{|l|c|}
\hline \multicolumn{1}{|c|}{ Nodo } & Effective Size (valores absolutos) \\
\hline 1.- Francisco Trujillo & 51.923 \\
\hline 2.- Francisco Rivas & 48.714 \\
\hline 3.- José Morales & 33.941 \\
\hline 4.- José Muñoz & 20.929 \\
\hline 5.- Valentín Valuarte & 17.000 \\
\hline 6.- Antonio De La Torre & 16.000 \\
\hline 7.-Santiago Marzan & 15.000 \\
\hline 8.- Francisco Silva & 14.867 \\
\hline 9.- Joaquín Vásquez & 14.867 \\
\hline 10. - Juan Molina & 14.000 \\
\hline 11.- José Terán & 13.857 \\
\hline 12.- Cecilio Alarcón & 13.209 \\
\hline 13.- Bernardo Ávila & 12.877 \\
\hline 14.- Simón Valenzuela & 12.561 \\
\hline 15.- José Latorre & 11.983 \\
\hline 16.- Pedro Jasnavar & 11.162 \\
\hline 17.- Felipe Contreras & 10.444 \\
\hline 18.- Juan José Mena & 10.429 \\
\hline 19.- Tadeo Contreras & 10.429 \\
\hline 20.- León Ramírez & 10.085 \\
\hline
\end{tabular}

Fuente: (ARNAD, FNR, volúmenes 19, 21-26, 28-39, 44-50). 1.526 documentos de venta de derechos de uso de tierras. Procesado en UCINET versión 6.658. 
El Cuadro 2 evidencia que los actores instalados en los 20 principales AE de esta red coincidieron con quienes obtuvieron mayor valor In-Degree, según corrobora el Cuadro 2 (existe un 70\% de coincidencias de nombres en ambos listados). El resultado permite detectar que los mayores brokers de esta red tuvieron la capacidad de construir AE comprando los derechos a uso de las tierras más cotizadas del sector a vendedores que participaban escasamente en este tipo de transacciones. En efecto, en las transacciones de los dos principales brokers del Cuadro 2, Francisco Rivas y Francisco Trujillo, se identifica una variedad amplia de significados respecto al contenido de los derechos de uso adquiridos. El hallazgo descubre a estos individuos asimilando progresivamente información variada sobre aquellos significados y gestionando esa información en diversas compras para obtener ventajas en la adquisición. ${ }^{12}$

La conducta mencionada ¿rindió frutos patrimoniales en el mediano plazo? Para enfrentar este último aspecto, se construyó la variable Progresión Patrimonial sobre la renta agrícola de los actores identificados en los dos cuadros anteriores. El primer análisis en esta dirección fue la identificación de los 28 actores que integraron los Cuadros 1 y 2 en los Catastros Agrícolas de 1833, 1852 y 1855 . El Cuadro 3 expresa el valor peso de la producción agrícola anual destinada al mercado, declarada por cada uno de los 28 sujetos. En seis casos se encontraron datos por individuo en los tres periodos y en otros siete en dos periodos. En las ocasiones en que el catastro no informó datos, se completó el Cuadro 3 con la cifra 12,3, con tal de evitar los datos sin información y, para posteriormente, lograr un análisis de la progresión patrimonial del conjunto. La elección consideró que el catastro registró solo agricultores que detentaban una renta anual equivalente o superior a 12,4 pesos. Por tanto, la cifra 12,3 representa una renta tipo del pequeño agricultor excluido del registro catastral.

Los resultados del Cuadro 3 testimonian que la mayoría de los registros correspondieron a rentas agrícolas relacionadas con la pequeña y mediana propiedad que no alcanzaron el mínimo de 12,4 pesos anuales. ${ }^{13}$ Adicionalmente, se constató una evolución general en el patrimonio agrícola del conjunto. De los 28 actores identificados, 17 declararon en 1855 una renta mayor a la registrada en 1833 y solo dos manifestaron un descenso en ambos periodos extremos. Aunque como se verá, esta progresión varió ostensiblemente si se analiza cada caso. Cabe considerar que esta variable se construyó sin considerar devaluación del peso, pues las décadas de 1830 a 1860 coincidieron con un estancamiento general en los precios de los bienes (Riveros, 1987, p. 270). 
Cuadro 3 - Renta en pesos de brokers.

Catastros Agrícolas de 1833, 1852 y 1855

\begin{tabular}{|c|c|c|c|}
\hline Brokers & 1833 & 1852 & 1855 \\
\hline Antonio De La Torre & 25 & 200 & 352 \\
\hline Asencio Medina & 18,6 & 50 & 125 \\
\hline Bernardo Ávila & 12,3 & 12,3 & 520 \\
\hline Cecilio Alarcón & 12,3 & 12,3 & 12,3 \\
\hline Felipe Contreras & 12,3 & 300 & 164 \\
\hline Francisco Rivas & 18,6 & 800 & 2000 \\
\hline Francisco Silva & 12,3 & 150 & 600 \\
\hline Francisco Trujillo & 12,3 & 25 & 250 \\
\hline Joaquín Vásquez & 12,3 & 12,3 & 12,3 \\
\hline José Latorre & 12,3 & 50 & 150 \\
\hline José Morales & 50 & 300 & 195 \\
\hline José Muñoz & 12,3 & 600 & 12,3 \\
\hline José Terán & 12,3 & 12,3 & 50 \\
\hline Juan José Mena & 12,4 & 12,3 & 12,3 \\
\hline Juan Molina & 25 & 250 & 270 \\
\hline León Ramírez & 12,4 & 12,3 & 12,3 \\
\hline Lorenzo Banda & 12,3 & 12,3 & 12,3 \\
\hline Pedro Jasnavar & 12,3 & 12,3 & 12,3 \\
\hline Pedro Lorca & 12,3 & 12,3 & 50 \\
\hline Ramón Jiménez & 12,3 & 100 & 144 \\
\hline Salvador Arellano & 12,3 & 12,3 & 12,3 \\
\hline Santiago Bosa & 12,3 & 12,3 & 100 \\
\hline Santiago Marzan & 12,3 & 100 & 50 \\
\hline Simón Valenzuela & 12,3 & 12,3 & 12,3 \\
\hline Tadeo Contreras & 12,3 & 12,3 & 12,3 \\
\hline Valentín Madariaga & 50 & 800 & 2500 \\
\hline Valentín Valuarte & 12,3 & 800 & 25 \\
\hline Victorino Guevara & 12,3 & 12,3 & 12,3 \\
\hline
\end{tabular}

Fuente: (Archivo Nacional Histórico, 1834; Oficina Central de Estadísticas, 1855a; 1855b). 
El cruce de las variables está representado en el Gráfico 1, en el cual cada una figura como vector. Destacan los nombres de los 17 actores que, tras la combinación de las tres variables, obtuvieron los valores resultantes más altos. El vértice donde converge el inicio de los vectores representa el promedio del valor de cada variable (situado, como se aprecia, en el cruce del respectivo valor 0 de los ejes Axis 1 y Axis 2 del gráfico). Por tanto, los sujetos ubicados a la derecha del vértice obtuvieron una cifra inferior a ese promedio, mientras que aquellos situados a la izquierda alcanzaron una cifra mayor. Los cosenos de los ángulos entre los vectores reflejan las correlaciones entre las variables. Es decir, la cercanía entre los vectores implica una fuerte relación directa, mientras que vectores perpendiculares indican nula relación entre ambas variables. Por último, el gráfico señala el valor que obtuvo cada actor sobre cada variable, de acuerdo a la posición del actor sobre la longitud del respectivo vector. Esto es, tras el trazo de una línea perpendicular del actor sobre el vector, a mayor longitud el punto de cruce en el vector, mayor valor obtuvo el actor sobre esa variable.

Gráfico 1 - Análisis multivariado In-degree, Effective Size y Progresión Patrimonial. Red de venta de derechos de uso de tierras. Rengo, Chile, 1832-1860

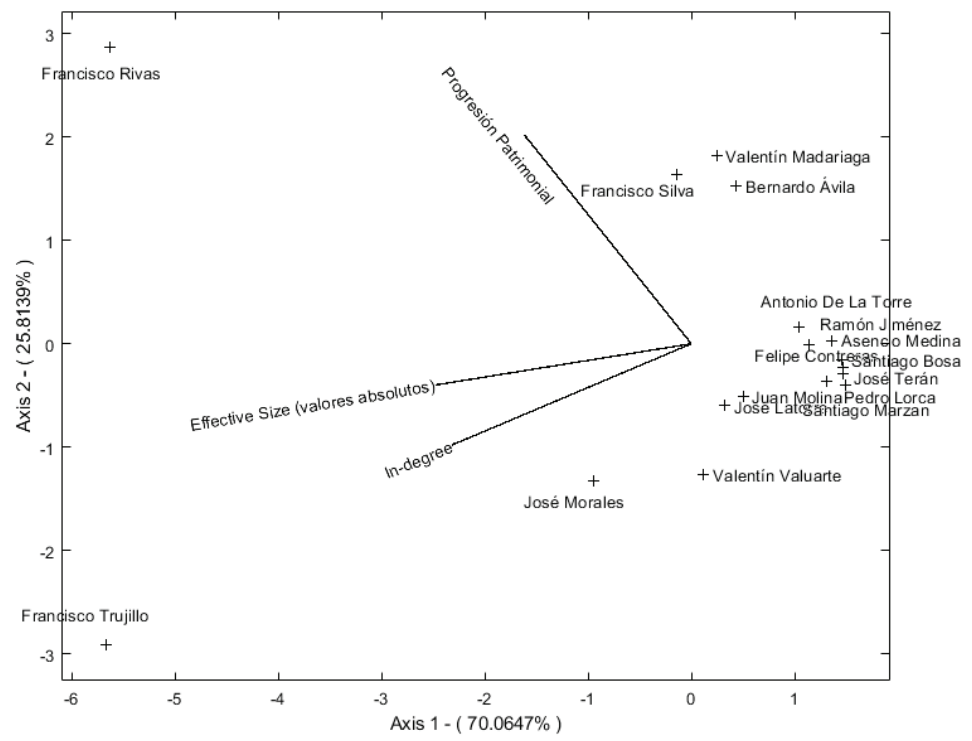

Fuente: (ARNAD, FNR, volúmenes 19, 21-26, 28-39, 44-50). 1.526 documentos de venta de derechos de uso de tierras (Archivo Nacional Histórico, 1834; Oficina Central de Estadísticas, 1855a; 1855b). 
El Gráfico 1 da cuenta de que las variables In-degree y Effective Size están fuertemente relacionadas entre sí (se encuentran próximas) y esta última mantiene una relación moderada con Progresión Patrimonial. Este resultado indica que la conducta de los principales brokers de esta red se basó en conseguir las tierras mejor valuadas, privilegiando transacciones con vendedores que participaron en escasa cantidad de ventas. Pero además, señala que esta conducta tuvo una repercusión moderada en su progresión patrimonial. De los 17 actores que figuran en el Gráfico 1, solo cuatro superaron el promedio de los valores integrados en las tres variables (a la izquierda del vértice de convergencia de los vectores): José Morales, Francisco Trujillo, Francisco Rivas y Francisco Silva. Fueron los cuatro actores de la muestra, cuya estrategia descrita de construcción de AE repercutió en el incremento de su renta agrícola. Pero los resultados difieren entre ellos. Francisco Rivas fue el caso de mayor éxito en Progresión Patrimonial con altos valores In-Degree y Effective Size (trazando una línea perpendicular hacia la proyección de cada uno de los tres vectores, cruza en un punto situado más a la derecha en la longitud de cada vector). ${ }^{14}$ Por su parte, Francisco Trujillo, presentando la conducta de mayor broker, obtuvo una posición más rezagada en Progresión Patrimonial, en comparación a los dos actores antes mencionados (evidencia un cruce perpendicular en un punto bajo de la longitud del vector Progresión Patrimonial). ${ }^{15}$ Los casos de Francisco Silva y José Morales, situados levemente por sobre el vértice del promedio en cada variable, no sobresalieron en ninguna de las tres.

\section{DISCUSIÓN: BROKERS EN EL CICLO DE FORMALIZACIÓN DE DERECHOS/DESPOSESIÓN CAMPESINA}

Los resultados del caso de estudio revelan la importancia de la gestión social de información sobre concepciones propietarias plurales, en periodo de formalización de derechos. Sobre todo, considerando los efectos de esa administración en la posición de los actores en la red de ventas y en su evolución patrimonial. Como se ha señalado anteriormente, la discusión ha puesto el acento en las concepciones propietarias plurales que convergieron en el siglo XIX latinoamericano (Barcos, 2013, p. 165). Desde el periodo colonial se heredaban interpretaciones y culturas jurídicas que asociaban la propiedad al cumplimiento del requisito de su función social concreta. Entonces, se esperaba que el beneficiario de la merced de tierra satisficiera las condiciones de residencia y producción. Sobre todo este último requisito fue acentuado en la 
región en el siglo XVIII, con el avance de las ideas productivistas y fisiócratas (Sánchez, 2019). Complementariamente resultaba válido el desmembramiento de los derechos de uso, goce y disposición del bien entre varios actores (Noejovich, 2003). En cambio, las legislaciones republicanas y las codificaciones impulsaron una concepción propietaria abstracta, en que esos tres derechos de dominio se concentraban en un propietario único y soberano sobre el bien (Escobar, A. et al., 2017). El enfoque basado en la convergencia de estos modelos propietarios ha enriquecido el modelo explicativo que señalaba la existencia de un ciclo de formalización de derechos sobre la tierra (en busca de la certificación de aquellos propietarios soberanos) seguido de un proceso de desposesión campesina. Se ha podido visualizar, en cambio, que distintos actores sociales lograban retener el acceso al uso o goce de la tierra, pese a quedar excluidos de la formalización de derechos. Se trataba de agricultores y poseedores de tierra que lograban gestionar en su favor este "haz de derechos" que se encontraban disponibles en un periodo de convergencia de concepciones propietarias (Poczynok, 2018).

El caso de estudio aproxima a los intentos de una muestra de agricultores, asociados a una rentabilidad media y baja, por adquirir los derechos de uso a tierra mejor valuada durante un periodo de formalización de derechos propietarios. Según se infiere del cruce de los Cuadros 1 y 2, por lo general los sujetos buscaron esa adquisición transando preferente con vendedores de baja participación en la red de venta de derechos de uso. ${ }^{16}$ De este modo, estos brokers se situaron en posiciones ejes de la estructura, reconocidas como AE según la propuesta de Ronald Burt (2010), concentrando variedad de concepciones propietarias que gestionaban con cierta ventaja en cada compra con sujetos aislados. Los datos contribuyen a la literatura subrayando la importancia de la posición que estos actores asumieron en estructuras relacionales que circularon concepciones propietarias plurales. Desde la gestión de esa posición, es posible observar los vaivenes en el acceso social a la tierra en periodo de formalización de derechos. Esta coyuntura crítica, reiterada en distintas latitudes de la región, no solo gatilló la restricción en el acceso a la tierra o la desposesión campesina, sino ha sido la tendencia a subrayar (LeGrand, 1988; Salazar y Pinto, 2002; Gelman y Santilli, 2013; Silva, 2015). También abrió una oportunidad para reconfigurar el tipo de redes sociales en las que los agricultores estaban inmersos hasta el momento, ensayando conductas relacionales que les redituaran frutos en el acceso a la tierra. Sobre todo en este escenario de coexistencia de concepciones propietarias plurales, en la que los actores podían gestionar la circulación de esa información heterogénea dentro de la red. 
Específicamente en relación con la literatura chilena, los resultados devienen significativos pues la discusión ha girado en torno al desarrollo de la gran propiedad como factor obstructivo en el acceso social a la tierra. Ya desde mediados del siglo XX, Jean Borde y Mario Góngora (1956) propusieron la rigidez e indivisión de la gran propiedad en el agro chileno entre 1680 y 1880, analizando un caso monográfico. El desarrollo progresivo del latifundio chileno, sobre todo en la zona central, había obstaculizado la circulación mercantil de tierras y propiciado la desposesión campesina (Salazar y Pinto, 2002; Cáceres, 2007).${ }^{17}$ Si bien últimamente, este modelo de latifundio rígido ha sido cuestionado por estudios más atentos a la división y reagrupación de la gran propiedad (Chuecas, 2018), no se cuenta con datos respecto al modo en que los actores experimentaron la convergencia de concepciones propietarias plurales, en coyuntura de formalización de derechos. En este sentido, el caso de estudio abre una oportunidad para profundizar las vías por las que, sujetos de distintos estratos sociales, administraron la coexistencia de nociones propietarias heterogéneas para obtener ventajas en el uso de la tierra. Resulta útil al respecto el primer resultado de este estudio. Los datos muestran que los sujetos aspiraron a la obtención de derechos de uso sobre la tierra mejor valuada transando con agricultores que no vendían estos derechos frecuentemente. Esta conducta aproxima a las expectativas de vendedores que participaban por primera vez en estos acuerdos y que no querían desprenderse del todo de sus derechos. La documentación refiere experiencias de compartición de derechos de uso postventa entre los contratantes, que explican la inclinación de los agricultores a vender a brokers experimentados en este tipo de acuerdos flexibles (remitimos al lector al ejemplo de la nota al pie de página número 9).

De todos modos, desde la perspectiva del logro de la conducta, el estudio de caso devela que la posición de broker en esta red no implicó necesariamente progresión patrimonial. Del conjunto de los 28 principales brokers, solo en cuatro existió una relación significativa entre aquella posición estructural y una mejora sostenida en la renta. Un acercamiento a los dos sujetos más sobresalientes, Francisco Rivas y Francisco Trujillo, evidenció que la mejora en sus rentas agrícolas se vincularon, además de su alta posición de broker en la red, a una participación alternada entre sus actividades agrícolas particulares y su vinculación con las esferas del poder administrativo local (sobre todo en el caso de Trujillo, que alcanzó la posición de alcalde y juez del municipio de Rengo). En este sentido, su progresión patrimonial también fue efecto de su participación en redes sociales de poder local, donde, por lo demás, tendían a ocupar posiciones axiales. Destaca el caso del agricultor Valentín Madariaga, 
quien no figuró como broker (no se encuentra en el Cuadro 2), pero apareció en el Cuadro 3 como uno de los principales ejemplos de progresión patrimonial. Este caso específico se explica por su posición central en la administración local. En 1836 fungió como gobernador interino del Departamento de Caupolicán y en 1838 alcanzó el puesto de alcalde de segunda elección del municipio de Rengo (Fondo Municipalidad de Rengo, vol. 1, fjs. 7, 8 y 11). Los resultados exhortan a la discusión a considerar la inserción de los sujetos en múltiples redes sociales para incrementar su capital social y económico. Este enfoque reconocido como "análisis múltiple" (Bright et al., 2015) ha rendido frutos en el escrutinio en perspectiva histórica, de estrategias de actores que obtuvieron recursos de su participación en redes sociales diversas. ${ }^{18}$ Una mirada de este tipo puede abrir campos de observación de alto interés en la problematización del modo en que los sujetos ligados a la tierra intentaron mantener o mejorar sus derechos al uso de este bien, durante el periodo de formalización de derechos y de revalorización mercantil de la tierra.

\section{Consideraciones FINALES}

Este estudio abordó la discusión que ha examinado el periodo de formalización de derechos y de restricción al acceso social a la tierra en Latinoamérica en el siglo XIX. El artículo se apoyó en el enfoque que ha complejizado la noción de propiedad, según el presupuesto de la negociación y disputa social sobre información plural relativa al derecho a la tierra. La interrogante giró en torno a las modalidades por las que los sujetos persiguieron sus intereses de acceso a la tierra, posicionándose en circuitos de compra y venta y gestionando nociones propietarias plurales. Se buscó analizar la conducta que asumieron los denominados brokers en una red de ventas de derechos de uso de tierra, en un valle de la zona central chilena, en periodo de formalización de derechos. Además, se pretendió evaluar los efectos a mediano plazo que tuvieron esas conductas en sus rentas. Metodológicamente se recurrió a la noción de AE, proveniente del Análisis de Redes Sociales, que permite detectar gestión de información plural por parte de nodos instalados en espacios de baja conexión en la red. Con estas herramientas y para abordar la interrogante y los objetivos propuestos, se analizaron tres variables: In-degree, Effective Size y Progresión Patrimonial (sometidas a un análisis multivariado), recurriendo a contratos contemporáneos de compra y venta de derechos de uso de la tierra y a tres catastros agrícolas.

Los resultados exhibieron una conducta tipo de los brokers, quienes pri- 
vilegiaron la compra a vendedores que contaban con baja experiencia en estas transacciones. Se trató de nodos que tuvieron la oportunidad de concentrar y distribuir información variada sobre los significados plurales respecto al contenido de los derechos de uso de la tierra. A través de esa gestión de información, abrían la posibilidad de adquirir los derechos a uso de las tierras mejor valuadas del valle. Sin embargo, los efectos patrimoniales de esta conducta fueron disímiles, pues solo en cuatro brokers se detectó progresión en la renta agrícola. El hallazgo contribuye a la literatura centrada en el proceso de formalización de derechos, complejizando el acceso social a los derechos sobre la tierra en esta coyuntura crítica. Los resultados exhortan a considerar la gestión que emprendieron sujetos interesados en la manutención y ampliación de sus derechos de uso sobre la tierra a partir de la administración de información plural, en escenarios en los cuales coexistieron concepciones propietarias heterogéneas. El caso de estudio visualiza la necesidad de complementar hallazgos de este tipo, indagando la participación de estos actores en redes sociales complementarias. Un "análisis múltiple" de esta índole aproximaría a los factores que condicionaron el éxito (o no) de la gestión de información variada sobre los derechos de uso de la tierra.

\section{Fuentes}

Archivo Nacional de la Administración de Chile. Fondo Notarios de Rengo, volúmenes 19, 21-26, 28-39, 44-50.

Archivo Nacional Histórico. Fondo de Contaduría Mayor, segunda serie. Catastro de la Provincia de Colchagua, volumen 3742, 1834.

Archivo Nacional Histórico. Fondo de Municipalidad de Rengo, volumen 1.

Oficina Central de Estadística. Rejistro jeneral del catastro formado el año de 1852. Santiago: Imprenta de J. Belín y Cía., 1855a.

Oficina Central de Estadística. Estadística Agrícola de Chile. Valparaíso: Imprenta del Diario La patria, 1855b.

\section{BibLIOGRAFÍA}

CORREA, Lily Álvarez. Catastro de propiedad en Chile: orígenes y evolución. Tesis (Doctorado en Geografía, Planificación Territorial y Gestión Ambiental) - Universidad de Barcelona. Barcelona, 2014. 
BARCOS, Maria Fernanda. Pueblos y ejidos de la campaña bonaerense. Una historia sociojurídica de los derechos de propiedad y la conformación de un partido: Mercedes, 1780-1870. Rosario: Prohistoria, 2013.

BORDE, Jean; GÓNGORA, Mario. Evolución de la propiedad rural del Valle del Puangue. Tomo I. Santiago: Instituto de Sociología, Universidad de Chile, 1956.

BORGATTI, Steven; EVERETT, Martin; JOHNSON, Jeffrey. Analyzing Social Network. New York: Sage, 2013.

BRANGIER, Víctor. Análisis de Redes Sociales y venta de tierras: un estudio de caso de Chile en perspectiva histórica. Población y Sociedad, La Pampa, v. 26, n. 1, p. 144-154, 2019.

BRIGHT, David et al. Networks within networks: using multiple link types to examine network structure and identify key actors in a drug trafficking operation. Global Crime, New York, v. 16, n. 3, p. 219-237, 2015.

BURT, Ronald. Structural holes versus network closure as social capital. In: Lin, N. et al. (eds.). Social capital: Theory and research. New York: Aldine de Gruyter, 2001. p. 31-56.

BURT, Ronald. Neighbor Networks. Competitive adventage local and personal. Oxford: Oxford University Press, 2010.

MUÑOZ, Juan Cáceres. Poder rural y estructura social. Colchagua, 1760-1860. La construcción del Estado y la ciudadanía desde la región. Valparaíso: Universidad Católica de Valparaíso, 2007.

CONGOST, Rosa; GELMAN, Jorge; SANTOS, Rui. (eds.). Property Rights in Land. Issues in social, economic and global history. New York: Routledge, 2017.

SALDÍAS, Ignacio Chuecas. Dueños de la Frontera. Terratenientes y sociedad colonial en la periferia chilena. Isla de Laja (1670-1845). Santiago: Biblioteca Nacional de Chile - Centro de Investigaciones Diego Barros Arana, 2018.

DEL VALLE, Laura Cristina; LARROSA, Juan. Actores y poder en el Cabildo del Buenos Aires, 1776-1810: una contribución desde el análisis de redes sociales. Revista Brasileira de História, São Paulo, vol. 39, n. 80, p. 135-154, 2019.

OHMSTEDE, Antonio Escobar et al. Introducción: En pos de las tierras civiles corporativas en México: la desamortización civil de la segunda mitad del siglo XIX. In: Escobar, A. et al. (org.). La desamortización civil desde perspectivas plurales. México: El Colegio de México - El Colegio de Michoacán - Ciesas, 2017. p. 11-65.

ESTADO DE CHILE. Códigos Chilenos. Leipzig: F.A. Brockhaus, 1882.

VILLARDON, Maria Purificación Galindo. Una alternativa de representación simultánea: HJ-Biplot. Questíio, Barcelona, vol. 10, n. 1, p. 13-23, 1986.

GARCÍA, Graciela Bonassa. Registros Paroquiais de terras. In: MOTTA, Márcia; GUIMARÃES, Elione (org.). Propiedades e Disputas: Fontes para História do Oitocentos. Guarapuava e Niterói: Unicentro-Eduff, 2011. p. 65-70. 
GELMAN, Jorge; SANTILLI, Daniel. Movilidad social y desigualdad en el Buenos Aires del siglo XIX: el acceso a la propiedad de la tierra entre el rosismo y el orden liberal. Hispanic American Historical Review, Durham, v. 93, n. 4, p. 659-684, 2013.

GROSSI, Paolo. La propiedad y las propiedades en el gabinete del historiador. Madrid: Civitas, 1992.

LATORA, Vito et al. Social cohesion, structural holes, and a tale of two measures. Journal of Statistical Physics, Kluwer Academic, v. 151, n. 3-4, p. 745-764, 2013.

LeGRAND, Catherine. Colonización y Protesta Campesina en Colombia, 1850-1950. Bogotá: Universidad Nacional de Colombia, 1988.

LLORCA-JAÑA, Manuel; NAVARRETE-MONTALVO, Juan. The Chilean Economy during the 1810-1830s and its Entry into the World Economy. Bulletin of Latin American Research, Blackwell Publishing, v. 36, n. 3, p. 354-369, 2017.

URQUIJO, José María Mariluz. El régimen de la tierra en el derecho indiano. Perrot, 1968.

NOEJOVICH, Hector Omar. La noción abstracta de propiedad en América: una visión desde los Andes. Derecho PUCP, v. 56, p. 989-1014, 2003.

PADGETT, John; ANSELL, Christopher. Robust Action and the Rise of the Medici, 1400-1434. American journal of sociology, v. 98, n. 6, p. 1259-1319, 1993.

PALACIOS, Marco. ¿De quién es la tierra? Propiedad, politización y protesta campesina en la década de 1930. Bogotá: FCE, Universidad de los Andes, 2011.

PISSIS, Pedro. Plano topográfico y geológico de la República de Chile. Paris: Chardon, 1873.

POCZYNOK, Cristian Miguel. La propiedad de la tierra como un "haz de derechos": una contribución desde la campaña bonaerense en un momento transicional (18001829). Mundo Agrario, La Plata, v. 19, n. 41, p. 1-20, 2018.

RIVEROS, Luis. Evolución de los precios en el siglo XIX. Estudios Públicos, Santiago, v. 27, p. 257-92, 1987.

SALAZAR, Gabriel; PINTO, Julio. Historia contemporánea de Chile III. La economía: mercados, empresarios y trabajadores. Santiago: LOM, 2002.

SAMUDIO, E. De propiedad comunal a propiedad individual en el escenario agrario republicano de Venezuela. El caso de Timotes. Mérida. Mundo Agrario, La Plata, v. 13, n. 25, p. 1-30, 2012.

SÁNCHEZ, Hugues. Entre la asignación de privilegios, el Estado y la causa pública: tierras y oratorio para el asiento de libres de Quilichao, Popayán, 1750-1810. Anuario Colombiano de Historia Social y de la Cultura, Bogotá, v. 46, n. 1, p. 59-83, 2019. SILVA, Marcio Antônio Both da. Land Law of 1850: Lessons on effects and the results of not condemn "a one fifth part of current agricultural population". Revista Brasileira de História, v. 35, n. 70, p. 87-107, 2015. 


\section{Notas}

${ }^{1}$ Este artículo es resultado de la investigación desarrollada en el marco del proyecto CONICYT/FONDECYT de Iniciación n. 11160130, periodo 2016-2019, del que el autor fue Investigador Responsable. El coautor integra la red HISONET (History \& Social Network), http://centroestudioshistoricos.ubo.cl/internacionalizacion/,derivada del proyecto anterior y realizó el análisis cuantitativo de los datos. Los autores agradecen a los evaluadores por sus comentarios que permitieron enriquecer la versión original del artículo.

${ }^{2}$ La Ley de Tierras de Brasil en 1850 fue uno de los ejemplos de mayor impacto. En los hechos, la legislación contribuyó a la informalización de posesiones, despojando campesinos de sus medios de subsistencia e incentivando su conversión en fuerza laboral dependiente en las grandes fincas de café (Silva, 2015). De todos modos, la ley también permitió su uso social. Ejemplo de ello son los registros paroquiais que desde 1854 obligaron a los poseedores a declarar sus tierras. Como no se obligó a comprobar la información, la práctica registral propició estrategias de agricultores para defender distintas formas de apropiación (García, 2011). En Colombia las leyes de tierras baldías de 1874 y 1882 habían generado un efecto similar (LeGrand, 1988, p. 37). Una síntesis de los efectos de políticas análogas sobre el acceso a la tierra en la campaña bonaerense se encuentra en: (Gelman y Santilli, 2013). El caso chileno sobre "descampesinización" puede consultarse en: (Salazar y Pinto, 2002, p. 97-99).

${ }^{3}$ Como recordó Héctor Noejovich (2003, p. 994), el derecho a propiedad se desmembra convencionalmente en estos tres poderes, reconocidos en la dogmática como ius utendi, ius fruendi y ius abutendi. En este sentido, ha resultado influyente la obra de Paolo Grossi (1992), quien avanzó una genealogía de conceptos jurídicos como "dominio útil", surgido desde el protagonismo del uso efectivo de la tierra en el medioevo. Esta sería una tradición milenaria jurídico-social que intentó quebrar el code civil napoleónico y la pandectística alemana, impulsando nociones abstractas de propiedad.

${ }^{4}$ Recientemente, Poczynok (2018) ofreció una síntesis bibliográfica para el caso de la campaña bonaerense. Una revisión del contexto venezolano se encuentra en: (Samudio, 2012).

${ }^{5}$ Según la historiadora argentina María F. Barcos (2013: p. 165), la discusión ha propiciado una aproximación a las tensiones entre "diferentes concepciones de la propiedad y diferentes prácticas propietarias".

${ }^{6}$ En la cultura política y jurídica de los dominios hispanoamericanos, la concepción de propiedad no resultaba abstracta, sino que tenía un anclaje concreto a su función social-productiva: se esperaba que el beneficiario de las mercedes de tierra otorgadas en nombre del rey cumpliera el requisito de residencia y trabajo (Mariluz, 1978). La corona cedía el goce de la tierra bajo condición de uso. Fue una noción que debió lidiar en el XIX con la emergencia de una concepción propietaria más abstracta, que aspiraba a la generación de propietarios con la facultad de usar, gozar y disponer soberanamente de sus bienes. Una síntesis de la literatura que ha abordado esta colisión de concepciones en América Latina en el siglo XIX, se puede consultar en (Escobar, A. et. al., 2017). 
${ }^{7}$ La primera vertiente de la discusión presupone que los sujetos obtienen mejores réditos al estar ubicados en redes cohesionadas. Estos escenarios les proporcionarían identidad, favoreciendo conductas colaborativas y reproducción de normas. La segunda corriente, por el contrario, propugna los beneficios de ubicarse en medio de redes abiertas, ricas en desconexiones y con vínculos débiles entre actores. Una síntesis del debate en: (Latora, et.al., 2013). La historiografía hispanoamericana sobre redes sociales coloniales se ha inclinado por el primer presupuesto, subrayando el poder reproducido por las élites tras la generación de redes familiares compactas (Del Valle y Larrosa, 2019).

${ }^{8}$ Respecto al "desmebramiento" de los derechos de dominio en Hispanoamérica (Mariluz, 1978; Noejovich, 2003).

${ }^{9}$ Por ejemplo, en 1832, el agricultor Santiago Valdovinos vendió en 90 pesos los derechos de uso a una porción de tierra en el valle de Rengo a su vecino colindante Manuel Guajardo. El contrato de venta indicaba que Guajardo debía "reservar el derecho q.e siempre a tenido [Rosa Valdovinos, hermana del vendedor] de cosechar las manzanas q.e dan los arboles" del terreno vendido. Notarios de Rengo, vol. 19, 17 de noviembre de 1832, fj. 39.

${ }^{10}$ Por lo general, los contratos de venta garantizaban estas expectativas de exclusión de derechos que adquirían los compradores, incluyendo fórmulas como: “... se desisten los vendedores quitar i apartar del derecho accion i dominio q.e tenian en la estancia vendida, i todo lo seden, renuncian i traspasan en el comprador...”. Notarios de Rengo, vol. 39, 3 de enero de 1850 , fj. 2.

${ }^{11}$ Por ejemplo, en agosto de 1837, el agricultor José Mena compró a Manuel, Cecilio, José, María, Juan y Tiburcio Mena, los derechos de uso de un cuarto de cuadra en 180 pesos. La transacción, que fijó el valor cuadra en 720 pesos, superó en más de $800 \%$ el promedio valor cuadra detectado en la muestra $(87,12$ pesos). En este contrato se explicitó que la compra incluía "todo lo edificado y plantado" (ARNAD, vol. 27).

${ }^{12}$ En julio de 1834, Francisco Rivas compró a su vecino colindante Gilberto Lazo los derechos de uso de media cuadra de tierras. Sin embargo, se especificó que el comprador se haría cargo de la reparación del cerco que mediaba entre los terrenos de ambos y que se encontraba en mal estado (Notarios de Rengo, vol. 19, 17 de julio de 1834). En cambio, en 1839, Rivas compró los derechos de ocho cuadras a Rosauro Gallegos, también vecina colindante. En esta ocasión el contrato señaló que la adquisición incluía la respectiva pared que separaba los predios (Notarios de Rengo, vol. 28, 19 de febrero de 1839). Del mismo modo, las compras de Francisco Trujillo develan mejoras progresivas en los términos de adquisición. De sus 49 compras, 30 las realizó en la década de 1850. En estas, a diferencia de las registradas en la década anterior, los derechos de uso adquiridos incorporaron el derecho a cursos de agua. Por ejemplo: (Notarios de Rengo, vol. 47, 14 abril 1852; vol. 49, 2 noviembre 1853 ).

${ }^{13}$ Las rentas declaradas por los brokers de este estudio se encuentran muy bajo el promedio de las rentas registradas para el valle de Rengo por los catastros de 1833 y 1852 (el catastro de 1855 no identifica las propiedades específicas del valle, sino del Departamento de Caupolicán, la unidad administrativa mayor). Mientras que el catastro de 1833 arrojó una ren- 
ta promedio para el valle de 163 pesos, el Cuadro 3 manifestó un promedio de solo 16 pesos. En el catastro de 1852, el promedio de renta fue de 398 pesos y en el Cuadro 3 de 167 pesos (Archivo Nacional Histórico, 1834; Oficina Central de Estadísticas, 1855a).

${ }^{14}$ A Francisco Rivas se le encontró en 1846 integrando un grupo de vecinos que aportaron recursos a la policía de Rengo para arreglo de caminos (Gobernación de Caupolicán, vol. 4, 1846), y en 1848 el Gobierno del Departamento de Caupolicán lo nombró tasador exclusivo para el avalúo de los daños en el vecindario que dejó la crecida del río durante el invierno (Gobernación de Caupolicán, vol. 38, 1848). Su breve vinculación con la administración pública se detuvo, y en los años siguientes se concentró en la adquisición de los derechos de uso de tierras, como indica la documentación notarial.

${ }^{15}$ El Fondo Gobernación de Caupolicán contiene información sobre la participación intensa de Trujillo en cargos de la administración local. Entre 1833 y 1843 asumió como Tesorero municipal en Rengo (Gobernación de Caupolicán, 1833: v. 4; 1843: v. 22). Entre 1841 y 1845 fue alcalde y juez de primera instancia de esta villa (Gobernación de Caupolicán, 1841: v. 20; 1845: v. 20). En 1843 se hizo cargo de los puestos de Teniente de Ministros, de Administrador de Correos en el Departamento y Administración de especies estancadas en Rengo. Entre 1845 y 1846 fue subdelegado en Rengo (Gobernación de Caupolicán, 1846: v. 31). Desde la década de 1850 no hay datos respecto a su participación en la esfera administrativa local y coincide con los años en que las fuentes notariales le descubren comprando la mayoría de los derechos de uso.

${ }^{16}$ Aunque este fue el comportamiento tipo de los brokers, hubo situaciones excepcionales, como la de Juan José Mena que alcanzó el segundo lugar en el Cuadro 1, y en el Cuadro 2 quedó decimoctavo. El resultado devela el intento de adquisición de derechos de uso de tierras bien valuadas (Cuadro 1) sin adquirir una conducta intensa de broker (Cuadro 2). Por el contrario, Mena compró derechos a agricultores con participación frecuente en estas transacciones. El Cuadro 3 indica que esta estrategia alterna no rindió frutos en su progresión patrimonial, pues en los tres catastros manifestó la misma renta mínima.

${ }^{17}$ Desde la segunda mitad del siglo XX la historiografía rural en Chile se enfocó en el desarrollo del latifundio. En la década de 1980, el despliegue de la Historia Social Desde Abajo motivó el análisis de la "descampesinización" que generó la dilatación de la gran propiedad, sobre todo desde la segunda mitad del siglo XIX (Brangier, 2019).

${ }^{18}$ La referencia obligada al respecto sigue siendo el estudio de Padgett y Ansell (1993), quienes examinaron patrones matrimoniales de las principales familias florentinas renacentistas. 